\title{
Unusual presentation of familial Mediterranean fever: role of genetic diagnosis
}

\begin{abstract}
Ran Nir-Paz, Eldad Ben-Chetrit, Eli Pikarsky, David Hassin, Yonathan Hasin, Tova Chajek-Shaul
\end{abstract}

\begin{abstract}
Objective-To describe the role of molecular analysis in the diagnosis of an unusual presentation of familial Mediterranean fever (FMF).

Case report-Two patients presenting with prolonged fever without signs and symptoms of serositis are described. FMF was diagnosed by genetic analysis, which disclosed that both patients were homozygous for the M694V mutation of the Mediterranean fever (MEFV) gene.

Conclusion-Molecular analysis of FMF should complement the investigation of patients with fever of unknown origin. This test enables a definite diagnosis of the disease and may promote the diagnosis and treatment of patients with an unusual or incomplete clinical picture of FMF. (Ann Rheum Dis 2000;59:836-838)
\end{abstract}

Familial Mediterranean fever (FMF) is an autosomal recessive disease, characterised by recurrent attacks of fever and peritonitis, pleuritis, arthritis, or erysipelas-like skin disease. ${ }^{1}$ The disease occurs predominantly among Sephardic Jews, Armenians, Turks, and Arabs, though sporadic cases have been found among Ashkenazic Jews. ${ }^{2}$ A typical attack consists of fever and serositis, lasting from one to four days. Between attacks patients are free of symptoms and appear healthy. The frequency of attacks varies considerably from weekly bouts to once every three to four months, or even less. Symptoms of FMF appear in the first decade of life in about $50 \%$ of patients, and only $5 \%$ develop the disease after the age of $30 .^{13}$ The severity of the attacks and their frequency usually decrease throughout the person's lifespan.

Until recently, the diagnosis of FMF was based on clinical manifestations, ethnicity, family history, and response to colchicine. In cases where these components were atypical or unhelpful, FMF was difficult to diagnose. Recently, two independent consortia identified and isolated the gene responsible for FMF (MEFV) on the short arm of chromosome $16 .{ }^{45}$ Cloning of MEFV now allows a new and reliable diagnostic test for FMF. A set of polymerase chain reaction primers can be used to demonstrate the mutations responsible for the disease. This important diagnostic tool has become essential in the diagnosis of patients with FMF who have an atypical clinical course and manifestations.

During 1999 we encountered two patients with fever of unknown origin, who were eventually proved to have FMF. We discuss here the important role of molecular analysis in making the diagnosis in atypical presentations of FMF.

\section{Case reports}

PATIENT A

A 48 year old Jewish woman of Sephardic origin was referred because of three years of biweekly episodes of fever up to $39.5^{\circ} \mathrm{C}$. The patient was admitted several times to another hospital where she underwent a thorough examination, including bone scan, gallium scan, upper and lower gastrointestinal series followed by gastroscopy and colonoscopy, multiple total body computed tomographies, abdominal sonography, echocardiography, and multiple blood and urine cultures, as well as a thorough search for mycobacterium. The results of all tests were normal or sterile respectively, except for a finding of normocytic anaemia with normal serum iron levels, and hypercalcaemia related to raised parathyroid hormone. A trial of steroids for treatment of occult inflammatory disease was unsuccessful. Her past medical history showed that since childhood she had had painful and swollen heels, especially after stress. Physical findings showing mild mitral stenosis and regurgitation and mild aortic regurgitation suggested a possible diagnosis of rheumatic heart disease.

On the present admission the patient complained of fever and night sweats. She was in good general condition with mildly raised blood pressure. Physical examination was unremarkable except for a $2 / 6$ systolic murmur compatible with mitral regurgitation and mild lymphadenopathy. The patient repeated a complete examination for her fever of unknown origin, which included serological tests and cultures, liver biopsy, temporal artery biopsy, bone marrow biopsy, lymph node biopsy, bronchoscopy, abdominal arteriography, and several imaging studies. No major pathological finding was evident except for anaemia (haemoglobin $86 \mathrm{~g} / \mathrm{l}$ ) and a raised erythrocyte sedimentation rate $(90 \mathrm{~mm} / 1 \mathrm{st} \mathrm{h})$. The attacks of fever were controlled with daily administration of $60 \mathrm{mg}$ prednisone.

At this point we learnt that her brother had FMF, which was successfully treated with colchicine. Although the clinical picture of the patient was not suggestive of FMF, we performed a molecular analysis of her DNA looking for mutations in the MEFV gene. The results showed that the patient was homozygous for the M694V mutation. FMF was diagnosed and colchicine treatment started. 


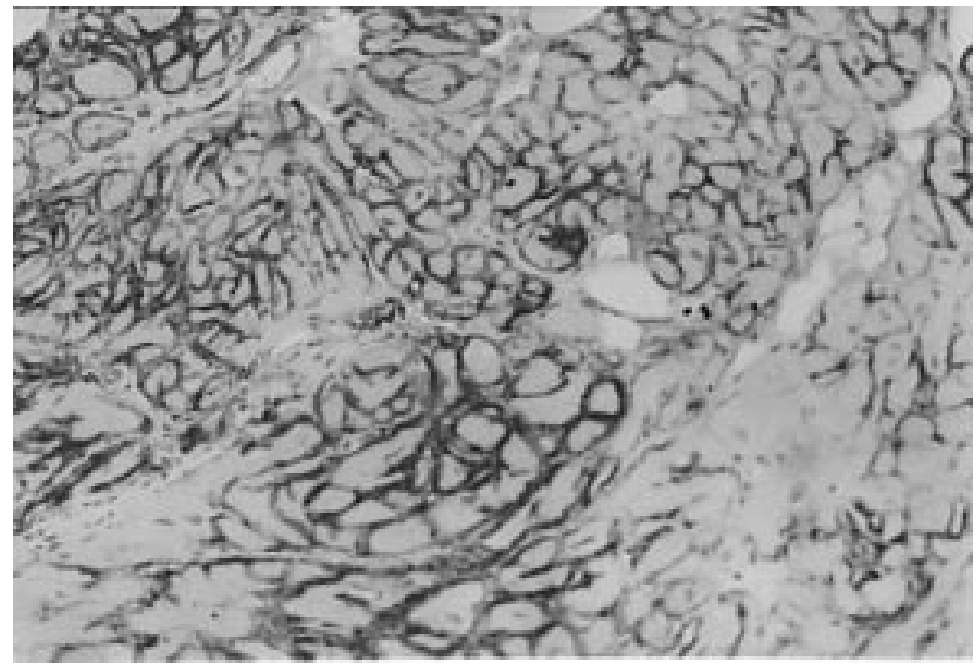

Figure 1 Endomyocardial biopsy specimen immunohistochemically stained with antibodies against amyloid $A$. The figure shows circumferential interstitial deposition of amyloid $A$ surrounding individual myocytes. Original magnification $\times 200$.

The patient took colchicine for about two months, but could not tolerate it. She had abdominal discomfort and diarrhoea even with a dose of $<1 \mathrm{mg}$ colchicine daily. She stopped the drug and continues to have episodic attacks of fever.

PATIENT B

A 54 year old Arab woman, born in the Lebanon, was referred to our department with heart failure of no apparent cause. She had been previously admitted to another hospital at the age of 32 years because of splenomegaly and proteinuria. Bone and liver biopsies were carried out, but the results were unavailable. Three years later a kidney biopsy was performed and showed focal glomerulonephritis with sclerosis. Since the age of 37 the patient had been readmitted many times owing to attacks of fever, cough, and weakness. Therapeutic trials with antibiotics for respiratory tract infection were partially effective. Computed tomography of the chest disclosed localised bronchiectasis in the left lower lobe of the lung. However, a gallium scan did not show any pathological emission. At the age of 40 treatment with steroids was started owing to a low level of antinuclear factor in the serum and suspicion of an underlying collagen vascular disease. The trial was unsuccessful and the fever attacks continued.

Re-evaluation of the patient's condition at the age of 50 showed several new findings, including symptoms and signs of left heart failure and the appearance of left bundle branch block in the electrocardiogram. Kidney, duodenal, and rectal biopsies showed sedimentation of amyloid fibrils around the blood vessels. An echocardiography performed during the present admission disclosed a mildly dilated left ventricle with global hypokinesis with regional variation (severe hypokinesis of septum and mild hypokinesis of the posterolateral wall); mildly to moderately reduced left ventricular function (left ventricular ejection fraction was $40-50 \%$ with no signs of a restrictive pattern); and wall thickness within the upper limits of normal. Cardiac catheterisation showed patent coronary arteries, moderately enlarged, and severely reduced contraction of the left ventricle. Repeated bacterial cultures (also for mycobacterium), and serological tests for infection or non-infectious inflammation, were normal or sterile, respectively. A myocardial biopsy showed deposition of amyloid A surrounding individual myocytes (fig 1). A diagnosis of chronic bronchiectasis with secondary amyloidosis was proposed. However, the ethnic origin of the patient, her clinical history of fever, and the finding of amyloidosis, raised the possibility of FMF. Indeed, molecular analysis showed that the patient was homozygous for the M694V mutation at the MEFV gene. Treatment with colchicine was started and the response was dramatic: the febrile attacks disappeared and the patient has a continuous feeling of wellbeing that she has not had for many years.

\section{Discussion}

Petersdorf and Beeson defined fever of unknown origin in 1961 as (a) temperatures higher than $38.3^{\circ} \mathrm{C}$ on several occasions; (b) duration of fever of more than three weeks; and (c) failure to reach a diagnosis despite one week of inpatient investigation. ${ }^{6}$

FMF is a disease in which fever is the main manifestation. However, the periodic nature of the disease in which the fever lasts up to 96 hours, and the relatively long interval between the attacks, are major criteria which assist in making the diagnosis of FMF. Furthermore, in contrast with most of the cases of fever of unknown origin, patients with FMF look completely healthy between the attacks. Therefore, the diagnosis of FMF is relatively easy in patients with typical clinical manifestations, family history, and appropriate ethnic origin. For that reason, in a large series of patients with fever of unknown origin, especially in those with a prolonged course of fever, only a few had a diagnosis of $\mathrm{FMF}^{78}$ As this diagnosis was made on clinical grounds only, it is still possible that even in these few cases the diagnosis was not correct. The recent isolation of germline mutations of the $55 \mathrm{kDa}$ tumour necrosis factor receptor causing a family of dominantly inherited autoinflammatory syndromes further emphasises the doubts and difficulties in clinical diagnosis of periodic fevers. ${ }^{9}$ Thus the relatively new diagnostic tool, molecular analysis, helps in making a definitive diagnosis of FMF in questionable or atypical cases.

The patients described herein both have appropriate ethnic origins as FMF is prevalent among North African Jews as well as among Arabs. ${ }^{12}$ However, their clinical presentations were unusual. The first patient had prolonged fever rather than periodic attacks. She did not complain of typical serositis. She had night sweats and lymphadenopathy, for which lymphoma was thoroughly searched. She was admitted to two hospitals and had undergone extensive clinical and laboratory investigations to exclude infection, malignancy, or collagen vascular disease, without any success. Because her brother was diagnosed as having FMF the 
patient underwent a molecular analysis. The test for detecting mutations responsible for FMF showed that she was homozygous for the M694V mutation. The lack of data about her response to colchicine due to her intolerance to this drug may raise some doubt about the diagnosis of FMF. However, the combination of molecular findings of homozygosity for the M694V mutation and the episodes of fever strongly indicate a diagnosis of FMF.

The second patient was transferred to our department for investigation of dilated cardiomyopathy. She had myocardial amyloidosis not usually seen in secondary amyloidosis that was thought to be secondary to bronchiectasis in a minor segment of her left lower lobe. However, molecular analysis disclosed that she was also homozygous for the M694V mutation. The question one may ask is whether this patient had more than one disease? We believe that this was not the case as, in retrospect, most of her complaints and findings could fit the diagnosis FMF, and she responded extremely well to colchicine treatment, though she had no family history of FMF. It is known that type II FMF phenotype presents with amyloidosis without preceding typical FMF attacks. The presence of amyloid in her myocardium is uncommon and probably reflects a disease course of many years with atypical presentation (dilated cardiomyopathy with no restrictive pattern). Such a pattern has not been reported before in FMF and is not a common consequence of bronchiectasis. ${ }^{10}$

Several studies comparing phenotype manifestations and genotype analysis have disclosed that patients with FMF homozygous for the M694V mutation have a more severe disease manifested by early onset, more frequent attacks, joint disease, and require higher doses of colchicine. ${ }^{11-13}$ Moreover, such patients are more prone to develop amyloidosis. ${ }^{13}$ More than 18 mutations of the MEFV gene are known, and studies have shown that some have low penetrance (E148Q, P396S), whereas others lead to a milder disease (V726A). ${ }^{13}{ }^{15} \mathrm{~A}$ few studies did not find similar results. ${ }^{16}$ Our experience is in accord with the former studies and we believe that the presence of the M694V mutation on both alleles in our patients strongly confirms the diagnosis of FMF.

We describe herein two unusual cases of fever of unknown origin due to FMF. Cases like these can mimic the presentation of other medical conditions, such as lymphoma, collagen vascular diseases, or infectious diseases. The presence of a prolonged benign course accompanied by amyloidosis, renal failure, and heart failure, should raise suspicions of FMF, especially in the absence of other causes of fever. The ability conclusively to diagnose or exclude FMF by a straightforward and reliable laboratory examination (that is, molecular analysis) changes the way we regard FMF. Nowadays, FMF should not be considered as a diagnosis of exclusion.

Nevertheless, we must still be patient and careful in using molecular analysis for diagnosis of FMF, especially in asymptomatic subjects as the complex issue of a genotype/ phenotype relation has not yet been settled.

We believe that, although rare, FMF should be included in the differential diagnosis of patients with fever of unknown origin, and molecular analysis for FMF should be complementary to their examination at some point. This treatable disease should not be left undiagnosed as missing the diagnosis can have severe consequences. Special efforts should be made to diagnose FMF, particularly when the ethnic background is appropriate.

1 Eliakim M, Levy M, Ehrenfeld M. Recurrent polyserositis (familial Mediterranean fever). Amsterdam: Elsevier/North Holland Biomedical, 1981.

2 Ben-Chetrit E, Levy M. Familial Mediterranean fever. Lancet 1998;351:659-64.

3 Livneh A, Langevitz P, Zemer D, Padeh S, Migdal A, Sohar $\mathrm{E}$, et al. The changing face of familial Mediterranean fever. Semin Arthritis Rheum 1996;26:612-27.

4 The International FMF Consortium. Ancient missense mutations in a new member of the RORet gene family are likely to cause familial Mediterranean fever. Cell 1997;90: 797-807.

5 The French Consortium. A candidate gene for familial Mediterranean fever. Nat Genet 1997;17:25-31.

6 Petersdorf RG, Beeson PS. Fever of unexplained origin. Medicine (Baltimore) 1961;40:1-30.

7 Knockaert DC, Dujardin KS, Bobbaers HJ. Long-term follow-up of patients with undiagnosed fever of unknown origin. Arch Intern Med 1996;152:618-20.

8 Aduan R. Prolonged fever of unknown origin. Clinical Research 1978;26:558A.

9 McDermott MF, Aksentijevich I, Galon J, McDermott EM, Ogunkolade W, Centola M, et al. Germline mutations in extracellular domains of the $55 \mathrm{kDa}$ TNF receptor, TNFR1, define a family of dominantly inherited autoinAlatory syndromes. Cell 1999,97:133-44.

10 Allen DC, Doherty CC. Sudden death in patients with amyloidosis of the cardiac conduction system. Br Heart J 1984; 51:293-6.

11 Dewalle M, Domingo C, Rosenbaum M, Ben-Chetrit E, Catton D, Bernot A, et al. Phenotype-genotype correlation in Jewish patients suffering from familial Mediterranean fever. Eur J Hum Genet 1998;6:95-7.

12 Shohat $M$, Magai N, Shohat $T$, Chen X, Dagan $T$, Mimoumi A, et al. Phenotype-genotype correlation in familial Mediterranean fever: evidence for an association between Met694Val and amyloidosis. Eur J Hum Genet 1999;7:287-92.

13 Cazeneuve C, Sarkisian T, Pecheux C, Dervichian M, Nedelec B, Reinert P, et al. MEFV-gene analysis in Armenian patients with familial Mediterranean fever: diagnostic value and unfavorable renal prognosis of the M694V homozygous genotype-genetic and therapeutic implications. Am J Hum Genet 1999;65:88-97.

14 Livneh A, Langevitz P, Shinar Y, Zaks N, Kastner DL, Pras $\mathrm{M}$, et al. MEFV mutation analysis in patients suffering from amyloidosis of familial Mediterranean fever. Amyloid 1999;6:1-6.

15 Aksentijevich I, Torosyan Y, Samuels J, Centola M, Pras E, Chae JJ, et al. Mutation and haplotype studies of familial Mediterranean fever reveal new ancestral relationship and evidence for a high carrier frequency with reduced penetrance in the Ashkenazi Jewish population. Am J Hum Genet 1999;64:946-62.

16 Akar N, Misiroglu M, Yalcinkaya F, Akar E, Cakar N, Tumer $\mathrm{N}$, et al. MEFV mutations in Turkish patients suffering from familial Mediterranean fever. Hum Mutat (online) 2000;15:118-19. 